\title{
Eigenstate thermalization hypothesis beyond standard indicators: Emergence of random-matrix behavior at small frequencies
}

\author{
Jonas Richter $\odot,{ }^{1, *}$ Anatoly Dymarsky, ${ }^{2,3,4, \dagger}$ Robin Steinigeweg ${ }^{\oplus},{ }^{1}$ and Jochen Gemmer ${ }^{1, \ddagger}$ \\ ${ }^{1}$ Department of Physics, University of Osnabrück, Osnabrück, Germany \\ ${ }^{2}$ Moscow Institute of Physics and Technology, 9 Institutskiy pereulok, Dolgoprudny, Russia \\ ${ }^{3}$ Skolkovo Institute of Science and Technology, Skolkovo Innovation Center, Moscow, Russia \\ ${ }^{4}$ Department of Physics, University of Kentucky, Lexington, Kentucky, USA
}

(Received 4 August 2020; accepted 29 September 2020; published 23 October 2020)

\begin{abstract}
Using numerical exact diagonalization, we study matrix elements of a local spin operator in the eigenbasis of two different nonintegrable quantum spin chains. Our emphasis is on the question to what extent local operators can be represented as random matrices and, in particular, to what extent matrix elements can be considered as uncorrelated. As a main result, we show that the eigenvalue distribution of band submatrices at a fixed energy density is a sensitive probe of the correlations between matrix elements. We find that, on the scales where the matrix elements are in a good agreement with all standard indicators of the eigenstate thermalization hypothesis, the eigenvalue distribution still exhibits clear signatures of the original operator, implying correlations between matrix elements. Moreover, we demonstrate that at much smaller energy scales, the eigenvalue distribution approximately assumes the universal semicircle shape, indicating transition to the random-matrix behavior, and in particular that matrix elements become uncorrelated.
\end{abstract}

DOI: 10.1103/PhysRevE.102.042127

\section{INTRODUCTION}

Questions of equilibration and thermalization in isolated quantum many-body systems have experienced an upsurge of interest both from the theoretical and the experimental side over the last decades [1-3]. In this context, the eigenstate thermalization hypothesis (ETH) has been established as a key concept to explain the emergence of thermodynamic behavior, by assuming a certain matrix structure of physical operators $\mathcal{O}$ in the eigenbasis of generic Hamiltonians $\mathcal{H}$ [4-6]. Specifically, let $\mathcal{O}_{m n}=\langle m|\mathcal{O}| n\rangle$ denote the matrix element of $\mathcal{O}$ within the eigenstates $|m\rangle$ and $|n\rangle$ of $\mathcal{H}$, then the ETH ansatz reads $[3,7]$

$$
\mathcal{O}_{m n}=O(\bar{E}) \delta_{m n}+\Omega^{-\frac{1}{2}}(\bar{E}) f(\bar{E}, \omega) r_{m n},
$$

where $\omega=E_{m}-E_{n}$ is the difference between the eigenenergies $E_{m}$ and $E_{n}$ with mean energy $\bar{E}=\left(E_{m}+E_{n}\right) / 2, O(\bar{E})$ and $f(\bar{E}, \omega)$ are smooth functions of their arguments, and $\Omega(\bar{E})$ is the density of states. Furthermore, the $r_{m n}=r_{n m}^{*}$ are conventionally assumed to be (pseudo-)random Gaussian variables with zero mean and unit variance. (For earlier work, see also Refs. [8,9].) While the ETH is an assumption and a formal proof is absent, its validity (including the Gaussian

\footnotetext{
*jonasrichter@uos.de

†a.dymarsky@uky.edu

†jgemmer@uos.de
}

Published by the American Physical Society under the terms of the Creative Commons Attribution 4.0 International license. Further distribution of this work must maintain attribution to the author(s) and the published article's title, journal citation, and DOI. distribution of the $r_{n m}$ ) has been numerically confirmed for a variety of models and observables [10-18]. Generally, the ETH is believed to hold for nonintegrable models and physical (for instance, spatially local) observables. In contrast, the ETH is violated in integrable systems due to their extensive number of integrals of motion [19], as well as in strongly disordered models which undergo a transition to a many-body localized phase in one dimension [20]. In these cases, the off-diagonal matrix elements $r_{n m}$ deviate from the Gaussian distribution [18,21]. In addition, models exhibiting a weaker violation of the ETH, such as, models featuring so-called "quantum scars," where rare less entangled states are embedded in an otherwise thermal spectrum, have recently attracted a significant amount of interest (see, e.g., Refs. [22,23]).

While the formulation of the ETH in Eq. (1) is conventional [3], it is to some degree incomplete with regard to the statistical properties of the $\mathcal{O}_{m n}$. Specifically, for a given $\mathcal{H}$ and $\mathcal{O}$, the matrix elements $\mathcal{O}_{m n}$ are predetermined, and therefore the notion of (pseudo-)randomness of the $r_{n m}$ needs to be carefully defined. In the spirit of the Bohigas-GiannoniSchmit conjecture [24], we here advocate the strongest point of view, that below a certain energy scale all statistical properties of the off-diagonal matrix elements would match those of a Gaussian random ensemble. The central question of this paper is therefore to what extent the matrix elements $\mathcal{O}_{m n}$ can be represented as independently drawn random numbers?

Clearly, all $\mathcal{O}_{m n}$ cannot be random in the strict sense as they are constrained by the fact that the observables have to obey various algebraic relations (e.g., $\mathcal{O}^{2}=\mathbb{1}$ in case of $\mathcal{O}$ being a Pauli matrix acting on an individual spin). Furthermore, correlations between the $r_{m n}$ are necessary to reproduce the growth of certain four-point correlation functions in chaotic systems [25-27]. Likewise, the consistency of relaxation dynamics in 
local systems also requires the $r_{m n}$ to be correlated [28]. We therefore arrive at the important conclusion that the onset of random-matrix behavior has to be limited to matrix elements $\mathcal{O}_{m n}$ within a certain energy window specified by the relevant energy scale $\Delta E_{\mathrm{RMT}}$.

In this paper, we test the ETH in the case of a local spin operator in the eigenbasis of the paradigmatic spin-1/2 XXZ chain, for which we break integrability by means of (i) an additional next-nearest neighbor interaction or (ii) a single-site magnetic field in the center of the chain. (See Refs. [29-32] for related studies of the ETH and the emergence of quantum chaos in these models.) Going beyond the "standard" indicators of the ETH, we particularly investigate the existence of the scale $\Delta E_{\mathrm{RMT}}$ below which random matrix theory (RMT) prevails. To this end, we establish the eigenvalue spectrum of $\mathcal{O}$ as a sensitive probe of the correlations between the $\mathcal{O}_{m n}$. While the spectrum of the full spin operator includes only two eigenvalues $\pm 1 / 2$, we particularly focus on the spectrum of band submatrices at a fixed energy density $\bar{E}$ where the $\mathcal{O}_{m n}$ are restricted to a narrow band $\left|E_{n}-E_{m}\right| \leqslant \omega_{c}$. For such band submatrices, we demonstrate that the $\mathcal{O}_{m n}$ are in convincing agreement with conventional indicators of the ETH in the following sense: (i) the diagonal matrix elements form a "smooth" function of energy $O(\bar{E})$, (ii) the off-diagonal matrix elements follow a Gaussian distribution with a variance $f^{2}(\bar{E}, \omega)$ that depends smoothly on the mean energy and respective energy difference, and (iii) the ratio between the variances of diagonal and off-diagonal elements for small $\omega$ takes on the value predicted by RMT. However, despite (i)-(iii) being satisfied, we find that the eigenvalues of the band submatrices for $\omega_{c}$ larger than a certain value $\Delta E_{\mathrm{RMT}}$ still exhibit clear signatures of the original operator, implying correlations between matrix elements. At the same time, when the bandwidth is sufficiently decreased, the spectrum takes on an approximately semicircular shape, marking the transition where genuine random-matrix behavior occurs.

\section{Outline and reader's guide}

While our main goal is to demonstrate the existence of the scale $\Delta E_{\mathrm{RMT}}$, this paper includes a detailed discussion of standard indicators of the ETH extensively studied in this context. A reader already familiar with numerical studies of the ETH, including Refs. [3-7,10-18], may go directly to the relevant sections concerned with the investigation of $\Delta E_{\mathrm{RMT}}$. This paper is structured as follows. In Sec. II we introduce the models and observables and describe our approach to study the ETH. In particular, the spectrum of band submatrices as a probe for the onset of random-matrix behavior is discussed in Sec. II B 3. Our numerical results are then presented in Sec. III. Specifically, we present data for "standard" indicators of the ETH in Sec. III A, while the main results concerning the existence of the scale $\Delta E_{\mathrm{RMT}}$ are analyzed in Sec. III B. A summary and discussion is given in Sec. IV, where we put our findings into context with previous studies of the ETH and outline future directions of research.

\section{SETUP}

\section{A. Models and observable}

In order to test the ETH ansatz (1), we consider different (integrable and nonintegrable) quantum spin chains. A

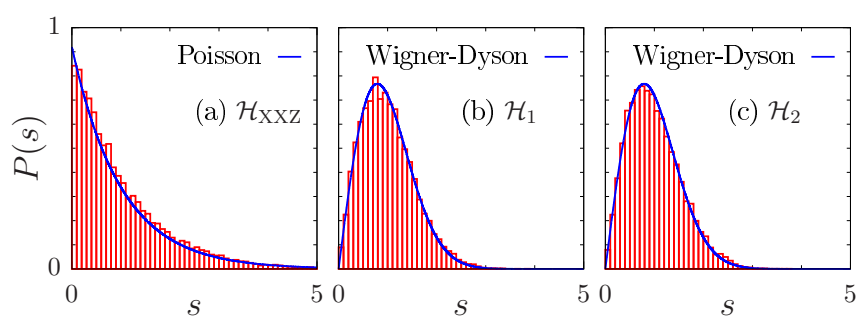

FIG. 1. Level-spacing distribution $P(s)$ of (a) $\mathcal{H}_{\mathrm{XXZ}}$, (b) $\mathcal{H}_{1}$, and (c) $\mathcal{H}_{2}$. The parameters are chosen as $\Delta=1.5, \Delta^{\prime}=1.2, h_{L / 2}=1$, and $L=18$. Correct extraction of $P(s)$ requires unfolding of the spectrum.

convenient starting point is provided by the one-dimensional XXZ model with open boundary conditions,

$$
\mathcal{H}_{\mathrm{XXZ}}=\sum_{\ell=1}^{L-1} S_{\ell}^{x} S_{\ell+1}^{x}+S_{\ell}^{y} S_{\ell+1}^{y}+\Delta S_{\ell}^{z} S_{\ell+1}^{z},
$$

where $L$ denotes the number of lattice sites, $S_{\ell}^{x, y, z}$ are spin-1/2 operators at site $\ell$, and $\Delta$ is an anisotropy in the $z$ direction. (In the following, we set the anisotropy to $\Delta=1.5$.) While $\mathcal{H}_{\mathrm{XXZ}}$ is integrable in terms of the Bethe ansatz, we break integrability by either an additional next-nearest neighbor interaction of strength $\Delta^{\prime}[17,33,34]$,

$$
\mathcal{H}_{1}=\mathcal{H}_{\mathrm{XXZ}}+\Delta^{\prime} \sum_{\ell=1}^{L-2} S_{\ell}^{z} S_{\ell+2}^{z},
$$

or by means of a single-site magnetic field $h_{L / 2}$ in the center of the chain [29-32,35,36],

$$
\mathcal{H}_{2}=\mathcal{H}_{\mathrm{XXZ}}+h_{L / 2} S_{L / 2}^{z} .
$$

Note that, although not written explicitly in Eqs. (2)-(4), we furthermore always include a small magnetic field at the first lattice site, $h_{1} S_{1}^{z}$ with $h_{1}=0.1$, which breaks the spin-flip and reflection symmetry of the model.

While $\mathcal{H}_{\mathrm{XXZ}}$ and $\mathcal{H}_{1,2}$ conserve the total magnetization $S^{z}=\sum_{\ell} S_{\ell}^{z}$, all results presented in this paper are obtained for the largest symmetry subspace which corresponds to $S^{z}=0$ and has dimension

$$
\mathcal{D}=\left(\begin{array}{c}
L \\
L / 2
\end{array}\right)=\frac{L !}{(L / 2) !(L / 2) !} .
$$

For $L=18$, which is the largest system size we can treat numerically, we have $\mathcal{D}=48620$. Moreover, our simulations are performed for a representative choice of the integrability-breaking parameters, i.e., $\Delta^{\prime}=1.2$ and $h_{L / 2}=1$, for which both $\mathcal{H}_{1}$ and $\mathcal{H}_{2}$ are robustly nonintegrable (see also Refs. [17,33-36] for other parameter choices).

The transition from the integrable XXZ chain to the nonintegrable models $\mathcal{H}_{1}$ and $\mathcal{H}_{2}$ can for instance be seen from the level-spacing distribution $P(s)$ which is shown in Fig. 1. While the level spacings follows the Poisson distribution in the integrable case, $P(s)$ matches the Wigner-Dyson distribution for $\mathcal{H}_{1,2}$. In this context, let us note that the field $h_{1}$ at the edge of the chain does not break integrability of $\mathcal{H}_{\mathrm{XXZ}}$ [35], while the single impurity $h_{L / 2}$ in the center of the chain induces the onset of chaos $[35,36]$. 


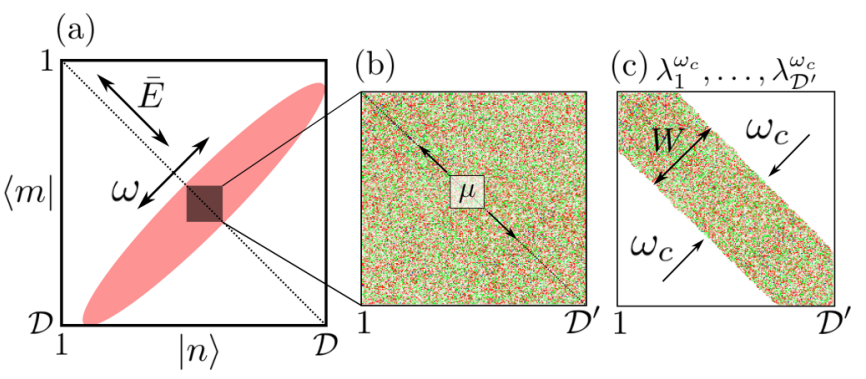

FIG. 2. (a) The ETH ansatz (1) is studied for the spin- $1 / 2$ operator $S_{L / 2}^{z}$ written in the eigenbasis of the respective Hamiltonian $\mathcal{H}$. The oval shaded area indicates matrix elements around a fixed value of $\bar{E}$ where the density of states is approximately constant, while the smaller square-shaped shaded area indicates a submatrix in this energy window. (b) For square-shaped submatrices with dimension $\mathcal{D}^{\prime}<\mathcal{D}$, the ratio $\Sigma^{2}(n, \mu)$ defined in Eq. (10) between the variances of diagonal and off-diagonal matrix elements is obtained for regions of size $\mu$ shifted along the diagonal. Note that the matrix shown here comprises actual data for the example of $\mathcal{H}_{1}$ and $L=12$. (c) We introduce a cutoff frequency $\omega_{c}$, where off-diagonal matrix elements are set to zero, $\mathcal{O}_{m n}=0$, if $\left|E_{m}-E_{n}\right|>\omega_{c}$, resulting in a band matrix with relative bandwidth $W / \mathcal{D}^{\prime}$. We study how the distribution of eigenvalues $\lambda_{1}^{\omega_{c}}, \ldots, \lambda_{\mathcal{D}^{\prime}}^{\omega_{c}}$ of the submatrix evolves upon reducing $\omega_{c}$.

For nonintegrable models such as $\mathcal{H}_{1,2}$, it is a general expectation that the matrix elements of physical observables $\mathcal{O}$ will follow the eigenstate thermalization hypothesis [3]. In this paper, we test the ETH for the case of a local spin-1/2 operator acting on the central lattice site of the chain,

$$
\mathcal{O}=S_{L / 2}^{z}
$$

Specifically, we employ full exact diagonalization to obtain the matrix elements $\mathcal{O}_{m n}$. Note that the indices $m$ and $n$ always refer to the eigenbasis of $\mathcal{H}$. The $\mathcal{O}_{m n}$ are real numbers for the chosen operator and the symmetry subspace.

\section{B. Testing the ETH and the onset of RMT}

In the following, we introduce the quantities studied in this paper. An accompanying sketch is provided in Fig. 2. A reader familiar with the "standard" indicators of the ETH may directly go to Sec. II B 3.

\section{Indicators of diagonal ETH}

The ETH ansatz (1) consists of the diagonal part and the off-diagonal part. The diagonal part of the ETH asserts that the function $O(\bar{E})$ becomes "smooth" in the thermodynamic limit $L \rightarrow \infty$. In particular, the eigenstate-to-eigenstate fluctuations $\mathcal{O}_{m m}-\mathcal{O}_{m+1 m+1}$ should rapidly decrease with the system size $L$. One way to test this statement is to study the variance $\sigma_{\mathrm{d}}^{2}(\bar{E})$ of the diagonal matrix elements,

$$
\sigma_{\mathrm{d}}^{2}(\bar{E})=\frac{1}{N_{\bar{E}}} \sum_{m}\left[\mathcal{O}_{m m}\right]^{2}-\left(\frac{1}{N_{\bar{E}}} \sum_{m} \mathcal{O}_{m m}\right)^{2}
$$

where the sum runs over all $N_{\bar{E}}$ eigenstates $|m\rangle$ with eigenenergies $E_{m} \in[\bar{E}-\Delta E / 2, \bar{E}+\Delta E / 2]$ in a microcanonical energy window around a fixed $\bar{E}$. For nonintegrable systems including our cases, it has been found that $\sigma_{\mathrm{d}}^{2}(\bar{E})$ decreases exponentially with increasing $L$, while the scaling for integrable models is polynomial; see, e.g., Refs. [3,11,17,37].

\section{Indicators of off-diagonal ETH}

Next, in order to test the off-diagonal part of the ETH, we consider matrix elements $\mathcal{O}_{m n}$ in a (sufficiently narrow) energy window around a fixed $\bar{E}$, where $\Omega(\bar{E})$ is approximately constant, which facilitates the analysis of the $\omega$ dependence of $f(\bar{E}, \omega)$ and of the distribution of the $\mathcal{O}_{m n}$; see Fig. 2(a). A useful quantity in this context is the average over matrix elements in a small $\omega$ interval, which we denote in this paper by an overline. For instance, the average over $\left|\mathcal{O}_{m n}\right|^{2}$ in an interval of width $\Delta \omega \ll \omega$ (with fixed $\bar{E}$ ) is given by

$$
\overline{\left|\mathcal{O}_{m n}\right|^{2}}(\omega)=\frac{1}{N_{\omega}} \sum_{\substack{n, m \\ E_{m}-E_{n} \approx \omega}}\left|\mathcal{O}_{m n}\right|^{2},
$$

where the sum runs over all $N_{\omega}$ matrix elements with $E_{m}-$ $E_{n} \in[\omega-\Delta \omega / 2, \omega+\Delta \omega / 2]$. Plotting $\overline{\left|\mathcal{O}_{m n}\right|^{2}}(\omega)$ versus $\omega$ yields the function $f^{2}(\bar{E}, \omega)$ [cf. Eq. (1)] except for an overall prefactor [18,38,39].

Assuming the $\mathcal{O}_{m n}$ have zero mean, i.e., $\overline{\mathcal{O}_{m n}}=0$, (which holds with a very high accuracy), we study the following quantity recently introduced in Ref. [18], which is sensitive to the distribution of $r_{n m}$,

$$
\Gamma(\omega)=\overline{\left|\mathcal{O}_{m n}\right|^{2}} /{\overline{\mid \mathcal{O}_{m n}}}^{2} .
$$

When $\overline{\mathcal{O}_{m n}}=0$, the nominator in Eq. (9) coincides with the variance of the $\mathcal{O}_{m n}$ while the denominator is the squared mean of the folded distribution. In particular, if $\mathcal{O}_{m n}$ were to follow the Gaussian distribution, $\Gamma(\omega)=\pi / 2$. The value we find numerically in Sec. III is very close. To further confirm that the distribution $P\left(\mathcal{O}_{m n}\right)$ of the $\mathcal{O}_{m n}$ is indeed Gaussian, we plot the histogram of $\mathcal{O}_{m n}$ from narrow windows with fixed $\bar{E}$ and $\omega$; see Fig. 5 below.

Next, we consider square-shaped submatrices of $\mathcal{O}$ of dimension $\mathcal{D}^{\prime}<\mathcal{D}$ around a fixed mean energy $\bar{E}$. In Fig. 2 (b), an example for such a submatrix comprising actual numerical data is shown. As a further check that the $\mathcal{O}_{m n}$ are normally distributed, we calculate the ratio $\Sigma^{2}(n, \mu)$ between the variances of diagonal and off-diagonal matrix elements for eigenstates in (small) regions $[n-\mu / 2, n+\mu / 2]$ of width $\mu$ [cf. Fig. 2(b)],

$$
\Sigma^{2}(n, \mu)=\frac{\sigma_{\mathrm{d}}^{2}(n, \mu)}{\sigma_{\text {od }}^{2}(n, \mu)} .
$$

Here $\sigma_{\mathrm{d}}^{2}(n, \mu)$ and $\sigma_{\text {od }}^{2}(n, \mu)$ are defined analogously to the variance in Eq. (7); see also Ref. [40] for details.

For an actual random matrix drawn from the Gaussian orthogonal ensemble (GOE), $\Sigma_{\mathrm{GOE}}^{2}=2$. Agreement with the GOE was anticipated in Ref. [3] and then verified numerically in, e.g., Refs. $[15,16,41]$. Our results for $\Sigma^{2}(n, \mu)$ in Sec. III are also in agreement with the GOE value.

\section{Indicators of correlations between off-diagonal matrix elements}

While the indicators of ETH given in Eqs. (7)-(10) have been studied before, this work particularly scrutinizes the 


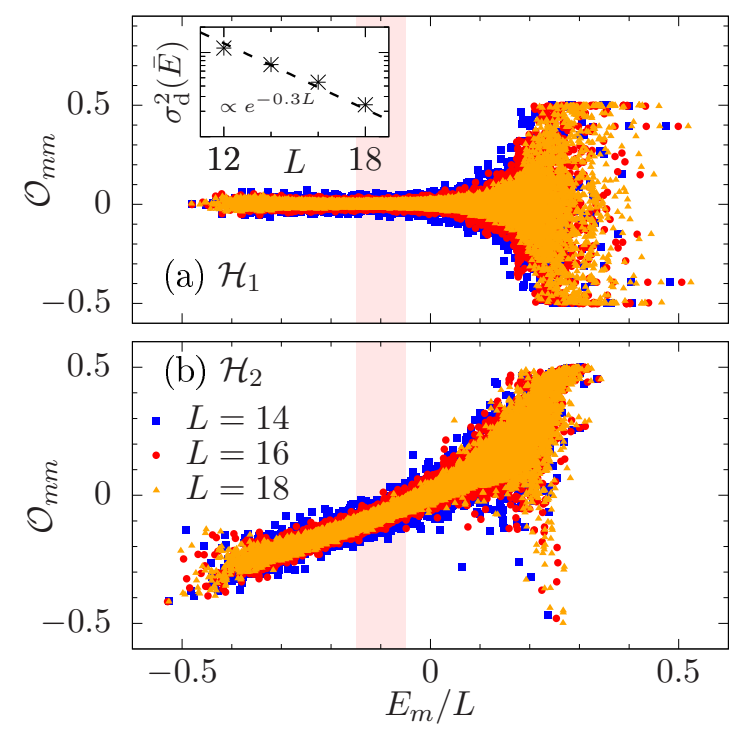

FIG. 3. Diagonal matrix elements of $S_{L / 2}^{z}$ in the eigenbasis of (a) $\mathcal{H}_{1}$ and (b) $\mathcal{H}_{2}$, for system sizes $L=14,16,18$. The shaded area indicates the energy window $E_{m} / L \in[-0.15,-0.05]$ which is used in the following to further study the properties of off-diagonal matrix elements. For the example of $\mathcal{H}_{1}$, the inset in (a) shows that the variance $\sigma_{\mathrm{d}}^{2}(\bar{E})$ of the $\mathcal{O}_{m m}$ in this window decreases exponentially with increasing $L$.

presence of correlations between the $\mathcal{O}_{m n}$. To this end, we consider the eigenvalue distribution of the submatrices with dimension $\mathcal{D}^{\prime}<\mathcal{D}$ around mean energy $\bar{E}$ [see Fig. 2(b)], and show that it provides a much more sensitive probe of the statistical properties of the $\mathcal{O}_{m n}$. For a full random matrix
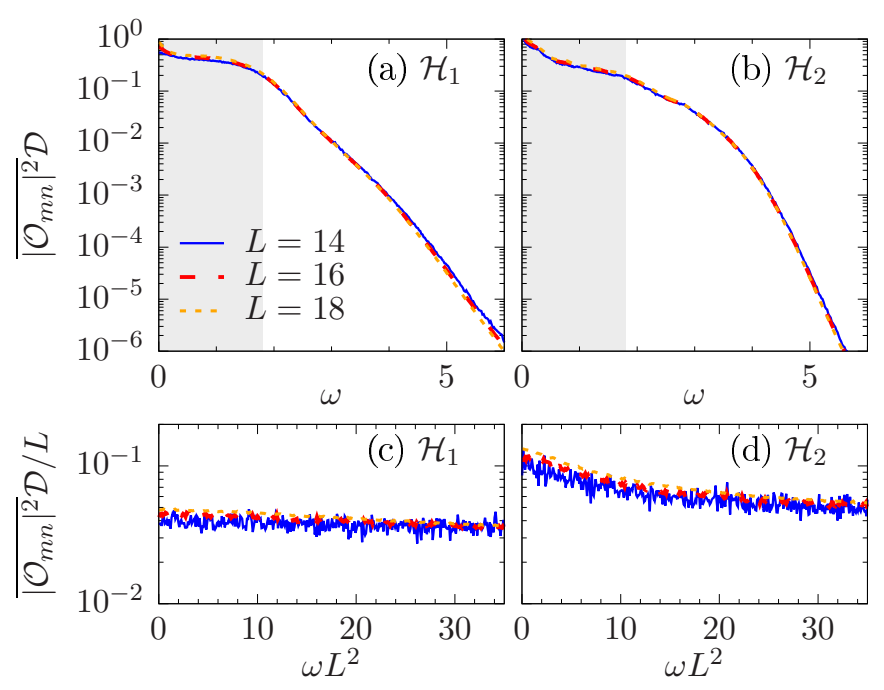

FIG. 4. [(a),(b)] Running averages $\overline{\left|\mathcal{O}_{m n}\right|^{2}}$ of matrix elements in the energy window $\bar{E} / L \in[-0.15,-0.05]$, calculated for system sizes $L=14,16,18$ and frequency bins of width $\Delta \omega=0.01$. (c, d) Close-up of the low-frequency regime using a bin width of $\Delta \omega=5 \times 10^{-4}$. Note that both the horizonal and the vertical axis have been rescaled. Panels (a) and (c) show results for $\mathcal{H}_{1}$, while panels (b) and (d) show data for $\mathcal{H}_{2}$. The shaded area in panels (a) and (b) indicates the $\omega$ range which is probed when considering a square-shaped submatrix with eigenstates in an energy interval of width $\Delta E / L=0.1$.
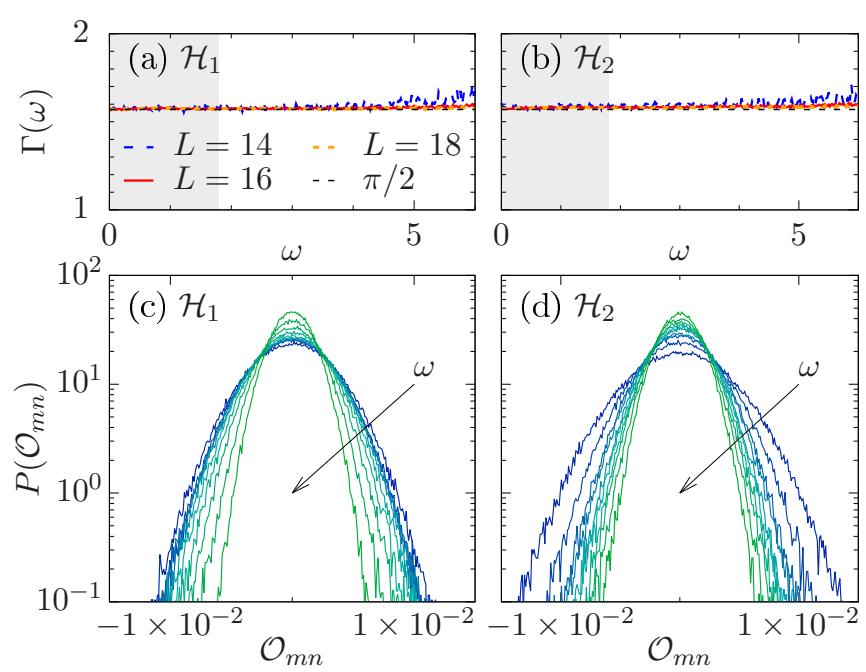

FIG. 5. [(a),(b)] $\Gamma(\omega)$ for matrix elements in the energy window $\bar{E} / L \in[-0.15,-0.05]$ and system sizes $L=14,16,18$. The dashed line indicates the value $\pi / 2$ for a Gaussian distribution. The shaded area indicates the $\omega$ range which is probed when considering a square-shaped submatrix with eigenstates in an energy interval of width $\Delta E / L=0.1$. (c, d) Distribution $P\left(\mathcal{O}_{m n}\right)$ of off-diagonal matrix elements for $L=18$ and $\omega=0.2,0.4, \ldots, 2$ (arrow). The data are collected in frequency bins $[\omega-\Delta \omega / 2, \omega+\Delta \omega / 2]$ with $\Delta \omega=0.002$. Panels (a) and (c) show results for $\mathcal{H}_{1}$, while panels (b) and (d) show data for $\mathcal{H}_{2}$.

with all matrix elements being independent, the eigenvalue distribution will follow the celebrated Wigner's semicircle $[3,42]$. In contrast, if there are correlations between the $\mathcal{O}_{m n}$, deviations from the semicircle shape should emerge. Importantly, we find that the eigenvalue spectrum unambiguously shows that the $r_{n m}$ can not be represented as independent Gaussians variables, even though all standard indicators of the ETH are fulfilled; see Sec. III B below. We note that this finding is in accord with recent theoretical arguments from Ref. [28]. In particular, Ref. [28] showed that consistency with transport in a quantum many-body system imposes constraints on the matrix elements entering the ETH and requires them to be correlated. The strongest constraint is provided by the slowest mode probed by the operator $\mathcal{O}$. For instance, consider a system exhibiting diffusive transport with $\mathcal{O}$ being coupled to the diffusive quantity (such a scenario is realized in the present paper as $\mathcal{O}=S_{L / 2}^{z}$ and spin transport is presumably diffusive in the nonintegrable models $\mathcal{H}_{1,2}$ [43]). In this case, the slowest Fourier mode is expected to decay as $\propto e^{-t / \tau}$ with $\tau \propto L^{2} / D$ and $D$ being the diffusion constant. While this picture would suggest that the $\mathcal{O}_{m n}$ should become stuctureless and independent for frequencies below $\tau^{-1} \propto L^{-2}$, Ref. [28] proved that the scale $\Delta E_{\mathrm{RMT}}$, below which genuine random-matrix may occur, in fact has to be parametrically smaller, $\Delta E_{\mathrm{RMT}} \lesssim(1 / \tau) / L \sim L^{-3}$. The bound $\Delta E_{\mathrm{RMT}} \lesssim(1 / \tau) / L$ with $\tau$ being the timescale of the slowest mode applies to any kind of transport. Therefore, in full generality, $\Delta E_{\mathrm{RMT}} \propto 1 / L^{2}$ is the loosest possible bound in a local system. Studying the signatures discussed below, we demonstrate in the present work that the scale $\Delta E_{\mathrm{RMT}}$ indeed 
exists. Moreover, while we do not explicitly address its scaling with $L$, we specifically show that $\Delta E_{\mathrm{RMT}}$ is drastically smaller than the scales where "standard" indicators of the ETH are already well fulfilled.

To identify the scale at which the transition to RMT behavior occurs, we analyze how the eigenvalue distribution depends on the width $W$ of the band; see Fig. 2(c). Specifically, let $\omega_{c}$ denote some cutoff frequency. Then we define the new operator $\mathcal{O}^{\omega_{c}}$ with matrix elements

$$
\mathcal{O}_{m n}^{\omega_{c}}= \begin{cases}\mathcal{O}_{m n}, & \left|E_{m}-E_{n}\right|<\omega_{c} \\ 0, & \text { otherwise }\end{cases}
$$

resulting in a band matrix with the relative bandwidth $W / \mathcal{D}^{\prime}$. Band random matrices have been extensively used in physics to model quantum systems and study their properties [44-49]. Furthermore, the largest eigenvalues of full (square) and band submatrices have been studied in Refs. $[28,41,50]$ in connection with the transition from integrability to chaos as well as relaxation dynamics and thermalization. However, to the best of our knowledge, the full eigenvalue distribution of (band) submatrices of local operators has not been previously considered as a quantity to characterize the presence of correlations between the matrix elements $\mathcal{O}_{m n}$. Provided all matrix elements are independent and identically distributed (except for an overall amplitude which may depend on $\omega$ ), the eigenvalue distribution of band random matrices is expected to converge towards a semicircle for small $W / \mathcal{D}^{\prime}$ [46], although there are corrections at intermediate $W / \mathcal{D}^{\prime}$ and the detailed shape is more complicated [51]; see Appendix C.

In addition to band submatrices, we also consider the eigenvalue distribution of full submatrices with varying dimension $\mathcal{D}^{\prime}$ in Appendix A. One advantage of keeping $\mathcal{D}^{\prime}$ fixed and varying $W$, however, is that the number of eigenvalues remains unchanged and is comparatively large. As shown in Appendix A, the properties of the smaller full submatrices are in fact similar and consistent with our findings for the band submatrices (11).

Given the ordered eigenvalues $\lambda_{\alpha}^{\omega_{c}}$ obtained by diagonalizing $\mathcal{O}^{\omega_{c}}$ for the cutoff frequency $\omega_{c}$, an important quantity characterizing $\mathcal{O}^{\omega_{c}}$ is the mean ratio $\left\langle r_{\omega_{c}}\right\rangle$ of adjacent level spacings,

$$
\left\langle r_{\omega_{c}}\right\rangle=\frac{1}{N_{r}} \sum_{\alpha} \frac{\min \left\{\Delta_{\alpha}, \Delta_{\alpha+1}\right\}}{\max \left\{\Delta_{\alpha}, \Delta_{\alpha+1}\right\}},
$$

where $\Delta_{\alpha}=\left|\lambda_{\alpha+1}^{\omega_{c}}-\lambda_{\alpha}^{\omega_{c}}\right|$ denotes the gap between two adjacent eigenvalues and the averaging is performed over a number (here $N_{r} \approx \mathcal{D}^{\prime} / 2$ ) of gaps around the center. For a random matrix drawn from the GOE, one expects $r_{\mathrm{GOE}} \approx$ 0.53 , while for uncorrelated Poisson distributed eigenvalues, one finds $r_{\text {Poisson }} \approx 0.39$ [52]. In addition to $\left\langle r_{\omega_{c}}\right\rangle$, the central quantity in this paper is the full eigenvalue distribution $P_{\omega_{c}}(\lambda)$ of the band submatrix $\mathcal{O}^{\omega_{c}}$,

$$
P_{\omega_{c}}(\lambda)=\frac{1}{\mathcal{D}^{\prime}} \sum_{\alpha=1}^{\mathcal{D}^{\prime}} \delta\left(\lambda-\lambda_{\alpha}^{\omega_{c}}\right),
$$

where $\delta(\cdot)$ denotes the $\delta$ function, and it is understood that individual peaks are collected in small bins such that $P_{\omega_{c}}(\lambda)$ forms a "continuous" distribution.
Given the corrections to the semicircle distribution for band random matrices with intermediate $W / D^{\prime}$, a particularly simple and effective scheme to test the randomness of $\mathcal{O}^{\omega_{c}}$ is to compare the eigenvalue distribution $P_{\omega_{c}}(\lambda)$ with the eigenvalue distribution of the suitably randomized $\widetilde{\mathcal{O}}^{\omega_{c}}$. For a similar comparison of the properties of bare and signrandomized matrices, see Refs. [53,54]. Specifically, $\widetilde{\mathcal{O}}^{\omega_{c}}$ is constructed by assigning random signs to the individual matrix elements $\mathcal{O}_{m n}^{\omega_{c}}$ (while keeping $\widetilde{\mathcal{O}}^{\omega_{c}}$ Hermitian),

$$
\widetilde{\mathcal{O}}_{m n}^{\omega_{c}}=\left\{\begin{array}{ll}
\mathcal{O}_{m n}^{\omega_{c}}, & 50 \% \text { probability } \\
(-1) \mathcal{O}_{m n}^{\omega_{c}}, & 50 \% \text { probability }
\end{array} .\right.
$$

If the matrix elements $\mathcal{O}_{n m}^{\omega_{c}}$ were random, we expect that the eigenvalue distribution would remain unchanged under this "sign randomization." In contrast, if the matrix elements of $\mathcal{O}^{\omega_{c}}$ are correlated, these correlations will be erased by the randomization procedure and the eigenvalue distribution of the original and randomized matrices will be different. In order to quantify the difference (and its dependence on $\omega_{c}$ ) between $P_{\omega_{c}}(\lambda)$ and the distribution $\widetilde{P}_{\omega_{c}}(\lambda)$ of the randomized operator, we introduce

$$
d_{2}\left(\omega_{c}\right)=\int_{-\infty}^{\infty}\left[P_{\omega_{c}}(\lambda)-\widetilde{P}_{\omega_{c}}(\lambda)\right]^{2} d \lambda,
$$

where $P_{\omega_{c}}(\lambda)$ and $\widetilde{P}_{\omega_{c}}(\lambda)$ should be understood as the continuous distributions resulting from a binning procedure [see below Eq. (13)]. If $d_{2}\left(\omega_{c}\right) \rightarrow 0$, both distributions are very similar, which will be interpreted as a further indication that the matrix elements $\mathcal{O}_{m n}^{\omega_{c}}$ are randomly distributed.

\section{RESULTS}

Let us now turn to our numerical results for the matrix structure of $S_{L / 2}^{z}$ in the eigenbasis of the two nonintegrable models $\mathcal{H}_{1}$ and $\mathcal{H}_{2}$. The properties of diagonal and off-diagonal matrix elements are discussed in Secs. III A 1 and III A 2, respectively, while Sec. III B presents results for the eigenvalue distribution of band submatrices. Additional results for the integrable $\mathrm{XXZ}$ chain can be found in Appendix B.

\section{A. "Standard" indicators of the ETH 1. Diagonal matrix elements}

As a first step, we study the diagonal part of the ETH. To this end, Figs. 3(a) and 3(b) show the matrix elements $\mathcal{O}_{m m}=$ $\left\langle m\left|S_{L / 2}^{z}\right| m\right\rangle$ as a function of the corresponding energy density $E_{m} / L$ for $\mathcal{H}_{1,2}$ and different system sizes $L=14,16,18$. For energy densities in the center of the spectrum, we find that the "cloud" of matrix elements becomes narrower with increasing $L[11,17,29]$. This finding is in good accord with the ETH prediction that the $\mathcal{O}_{m m}$ should form a "smooth" function of energy in the thermodynamic limit $L \rightarrow \infty$. At the edges of the spectrum, the scaling with $L$ is significantly slower. Especially for $\mathcal{H}_{1}$ [Fig. 3(a)] and $E_{m} / L \gtrsim 0.2$, the $\mathcal{O}_{m m}$ are found to fluctuate very strongly. This can be understood as follows. The eigenstates of $\mathcal{H}_{1}$ with the highest energies are weakly dressed domain-wall states. Consider, for instance, the states $\left|n_{1}\right\rangle=|\uparrow \cdots \uparrow \downarrow \cdots \downarrow\rangle$ and $\left|n_{2}\right\rangle=|\downarrow \cdots \downarrow \uparrow \cdots \uparrow\rangle$ (note that $\left|n_{1}\right\rangle$ and $\left|n_{2}\right\rangle$ are not exact eigenstates). 
While $\left|n_{1}\right\rangle$ and $\left|n_{2}\right\rangle$ have almost the same energy, one finds that $\left\langle n_{1}\left|S_{L / 2}^{z}\right| n_{1}\right\rangle \approx 1 / 2$ whereas $\left\langle n_{2}\left|S_{L / 2}^{z}\right| n_{2}\right\rangle \approx-1 / 2$ such that ETH is not satisfied. We expect the range of energy densities where the ETH applies to increase with $L$.

Given the distribution of the $\mathcal{O}_{m m}$, we restrict ourselves in the following to eigenstates in an energy window $E_{m} / L \in$ $[-0.15,-0.05]$ which is close to the center of the spectrum (shaded area in Fig. 3). As shown in the inset of Fig. 3(a), the variance $\sigma_{\mathrm{d}}^{2}(\bar{E})$ of the $\mathcal{O}_{m m}$ in this window decays approximately exponentially with $L$ (at least for the system sizes numerically available), indicating that the diagonal part of the ETH is fulfilled.

\section{Off-diagonal matrix elements}

Let us now analyze the properties of the off-diagonal matrix elements $\mathcal{O}_{m n}=\left\langle m\left|S_{L / 2}^{z}\right| n\right\rangle$. In view of the previous results for the diagonal matrix elements in Fig. 1, we focus on eigenstates with mean energy density $\bar{E} / L \in[-0.15,-0.05]$ as said above. For matrix elements in this window, running averages $\overline{\left|\mathcal{O}_{m n}\right|^{2}}$ of their absolute squares are shown in Figs. 4(a) and 4(b) as a function of $\omega$ both for $\mathcal{H}_{1}$ and $\mathcal{H}_{2}$. The data are obtained for frequency intervals of width $\Delta \omega=10^{-2}$ and system sizes $L=14,16,18$. Overall, the situation is qualitatively similar for the two models $\mathcal{H}_{1,2}$. Namely, $\overline{\left|\mathcal{O}_{m n}\right|^{2}}$ decays comparatively slowly at low frequencies, while a substantially quicker (presumably superexponential $[55,56]$ ) decay can be found at higher $\omega$. In Figs. 4(a) and 4(b) the values of $\overline{\left|\mathcal{O}_{m n}\right|^{2}}$ for different $L$ form smooth curve which collapse on each other when rescaled by the respective Hilbert-space dimension $\mathcal{D}$. [The rescaling by $\mathcal{D}$ accounts for the factor $\Omega^{-\frac{1}{2}}(\bar{E})$ in Eq. (1).] Except for a prefactor, these smooth curves correspond to the function $f^{2}(\bar{E}, \omega)$ from the ETH ansatz (1).

For a more detailed analysis of $\overline{\left|\mathcal{O}_{m n}\right|^{2}}$, Figs. 4(c) and 4(d) show a close-up of the low-frequency regime (note that the horizontal and the vertical axis have been rescaled to account for possible finite-size effects at such small $\omega$ ). For both $\mathcal{H}_{1}$ and $\mathcal{H}_{2}$, we observe that $\overline{\left|\mathcal{O}_{m n}\right|^{2}}$ clearly approaches a nonzero value as $\omega \rightarrow 0$ with an approximately constant plateau for small $\omega L^{2}$, where the data collapse achieved by the $L^{2}$ rescaling indicates diffusive spin dynamics (see also the discussion in Ref. [30]).

Next, in order to study the distribution of the $\mathcal{O}_{m n}$, Figs. 5(a) and 5(b) show the frequency-dependent ratio $\Gamma(\omega)$, defined in Eq. (9). For small $\omega$, we find that $\Gamma(\omega)$ is close to the Gaussian value $\pi / 2$, while visible deviations appear at higher frequencies. However, these deviations decrease with the increasing system size $L$, indicating that the $\mathcal{O}_{m n}$ follow a Gaussian distribution over a wide range of frequencies if $L$ is sufficiently large. In addition, the full distribution $P\left(\mathcal{O}_{m n}\right)$ of the off-diagonal matrix elements is shown in Figs. 5(c) and 5(d) for system size $L=18$ and frequencies $\omega=0.2,0.4, \ldots, 2$. For all curves shown, we find that $P\left(\mathcal{O}_{m n}\right)$ is indeed well described by the Gaussians with zero mean (see also Refs. [12,18,21]). The width of the Gaussians is found to decrease with increasing $\omega$, which is consistent with the data for $\overline{\left|\mathcal{O}_{m n}\right|^{2}}$ shown in Figs. 4(a) and 4(b).

Let us finally comment on the shaded gray area at low frequencies in Figs. 4(a) and 4(b) and Figs. 5(a) and 5(b). This area indicates the frequency range which is covered
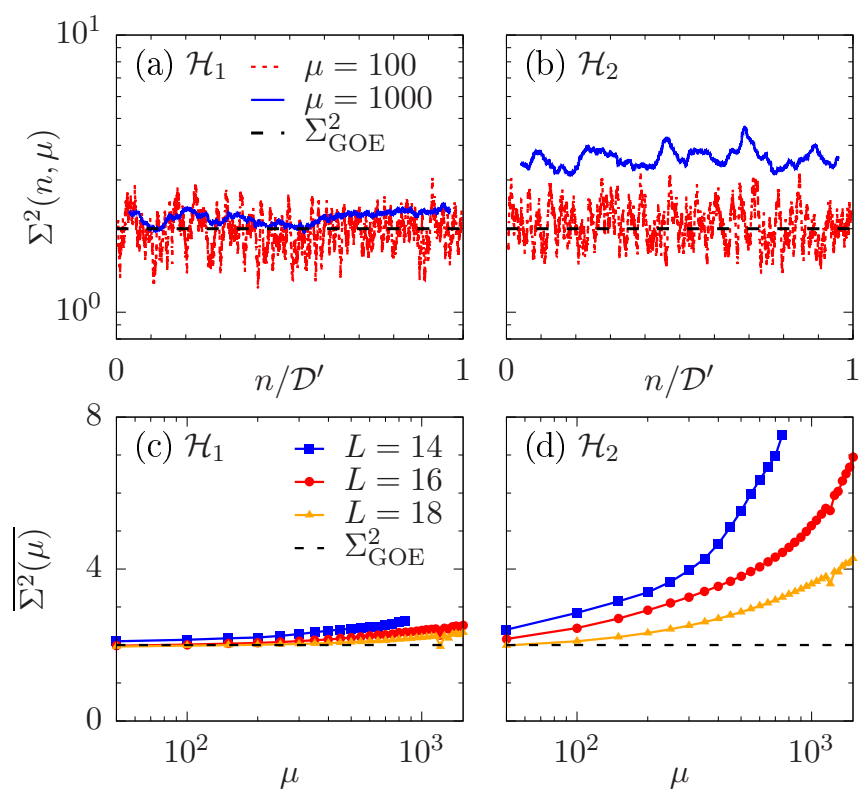

FIG. 6. [(a),(b)] Ratio $\Sigma^{2}(n, \mu)$ between the variances of diagonal and off-diagonal matrix elements for two different square sizes $\mu=100,1000$ and all embeddings $n \in\left[1+\mu / 2, \mathcal{D}^{\prime}-\mu / 2\right]$. The data are obtained for system size $L=18$ and the dashed line indicated the value $\Sigma_{\mathrm{GOE}}^{2}=2$ predicted from RMT. [(c),(d)] Average value $\overline{\Sigma^{2}(\mu)}$ versus $\mu$ for system sizes $L=14,16,18$. Note that for the largest system size $L=18$, the maximum $\mu=2 \times 10^{3}$ shown here is still considerably smaller than the full submatrix dimension $\mathcal{D}^{\prime} \approx 1.3 \times 10^{4}$. Panels (a) and (c) show data for $\mathcal{H}_{1}$ while panels (b) and (d) show data for $\mathcal{H}_{2}$.

when considering a square-shaped submatrix in the interval $\bar{E} / L \in[-0.15,-0.05]$; cf. Fig. 2. Specifically, since this interval has a width $\Delta E / L=0.1$, the largest energy difference for $L=18$ is $\omega_{\max }=0.1 L=1.8$. Therefore, when studying the eigenvalue distribution of such a submatrix further below, we are probing the region where $\overline{\left|\mathcal{O}_{m n}\right|^{2}}$ varies comparatively slowly and $\Gamma(\omega) \approx \pi / 2$.

To conclude the analysis of the off-diagonal matrix elements, Figs. 6(a) and 6(b) show the ratio $\Sigma^{2}(n, \mu)$ between the variances of diagonal and off-diagonal matrix elements. Specifically, the data are obtained for $L=18$ with two different square sizes $\mu=100,1000$ and all possible embeddings along the diagonal of the submatrix with dimension $\mathcal{D}^{\prime}$. (Note that for the chosen energy window, we have $\mathcal{D}^{\prime} \approx \mathcal{D} / 4$.) For small $\mu=100$, we find that $\Sigma^{2}(n, \mu)$ fluctuates around the GOE value $\Sigma_{\mathrm{GOE}}^{2}=2$, both for $\mathcal{H}_{1}$ and $\mathcal{H}_{2}$. Increasing the square size to $\mu=1000$, we observe that the fluctuations of $\Sigma^{2}(n, \mu)$ are visibly reduced. For the larger value of $\mu$, $\Sigma^{2}(n, \mu)$ is still rather close to $\Sigma_{\mathrm{GOE}}^{2}$ for $\mathcal{H}_{1}$, while clear deviations between $\Sigma^{2}(n, \mu)$ and $\Sigma_{\mathrm{GOE}}^{2}$ can be seen in the case of $\mathcal{H}_{2}$.

Figures 6(c) and 6(d) show the averaged value,

$$
\overline{\Sigma^{2}(\mu)}=\frac{1}{\mathcal{D}^{\prime}-\mu} \sum_{n=1+\mu / 2}^{\mathcal{D}^{\prime}-\mu / 2} \Sigma^{2}(n, \mu),
$$

calculated from all embeddings $n \in\left[1+\mu / 2, \mathcal{D}^{\prime}-\mu / 2\right]$. We find that $\overline{\Sigma^{2}(\mu)} \approx \Sigma_{\mathrm{GOE}}^{2}$ for small $\mu$, while $\overline{\Sigma^{2}(\mu)}$ 
monotonously grows with increasing $\mu$ (this growth is particularly pronounced in the case of $\mathcal{H}_{2}$ ). This behavior of $\overline{\Sigma^{2}(\mu)}$ follows from the $\omega$ dependence of $\overline{\left|\mathcal{O}_{m n}\right|^{2}}$ shown in Fig. 4. Since $\overline{\left|\mathcal{O}_{m n}\right|^{2}}$ decreases with increasing $\omega$, the variance $\sigma_{\text {od }}^{2}(n, \mu)$ likewise decreases with increasing $\mu$, simply because matrix elements at higher frequencies are included. Comparing $\overline{\Sigma^{2}(\mu)}$ for different system sizes, we find that $\overline{\Sigma^{2}(\mu)}$ remains closer to $\Sigma_{\mathrm{GOE}}^{2}$ for a wider range of $\mu$ as $L$ increases (see also Ref. [16]). Therefore in the thermodynamic limit $L \rightarrow \infty$ one can expect $\mathcal{O}_{m n}$ to approach an actual random matrix drawn from the GOE, at least for a finite region around the diagonal.

To summarize, in this subsection we considered different quantities conventionally considered as standard indicators of ETH. The results presented in Figs. 3-6 confirm that the matrix structure of the local spin-1/2 operator $\mathcal{O}=S_{L / 2}^{z}$ in the eigenbasis of the nonintegrable models $\mathcal{H}_{1,2}$ is in good agreement with the ETH ansatz (1), at least for the chosen energy window close to the center of the spectrum. Nevertheless, in the next subsection, we will show that the matrix elements $\mathcal{O}_{m n}$ within this energy window can not be considered as fully uncorrelated, i.e., the standard indicators in Figs. 3-6 are not sufficient when it comes to the statistical properties of the $\mathcal{O}_{m n}$.

\section{B. Beyond "standard" indicators: Eigenvalue distribution of band submatrices}

We now turn to the eigenvalue distribution for the band submatrices centered around $\bar{E} / L=-0.1$ with $\Delta E / L=0.1$. First, we discuss the ratio of the adjacent level spacings $\left\langle r_{\omega_{c}}\right\rangle$ defined in (12), which is shown in Fig. 7 versus $W^{2} / \mathcal{D}^{\prime}$ [Figs. 7(a) and 7(b)] as well as versus $\omega_{c}$ [Figs. 7(c) and 7(d)]. We find that the behavior of $\left\langle r_{\omega_{c}}\right\rangle$ is very similar for $\mathcal{H}_{1}$ and $\mathcal{H}_{2}$. Specifically, over a wide range of $\omega_{c},\left\langle r_{\omega_{c}}\right\rangle \approx 0.53$ approximately matches the GOE value, while the transition towards the Poissonian value $\left\langle r_{\omega_{c}}\right\rangle \approx 0.39$ occurs when the bandwidth becomes too narrow. The crossover from $r_{\mathrm{GOE}}$ to $r_{\text {Poisson }}$ can be understood from the well-known fact that the eigenstates of a band random matrix with a sufficiently small value of $W^{2} / D^{\prime}$ are localized and the eigenvalues become uncorrelated [45]. In order to avoid localization effects while studying the eigenvalue distribution $P_{\omega_{c}}(\lambda)$, we restrict our analysis to $\omega_{c} \gtrsim 0.03$ (shaded area in Fig. 7), such that $\left\langle r_{\omega_{c}}\right\rangle \approx r_{\mathrm{GOE}}$.

In Fig. 8 we show the full spectrum $P_{\omega_{c}}(\lambda)$ for $\mathcal{H}_{1,2}$ with $L=18$ and four exemplary choices of the cutoff frequency $\omega_{c}$. Specifically, we have chosen $\omega_{c}=1.8$ (i.e., the full nonband submatrix), as well as $\omega_{c} \approx 1, \omega_{c} \approx 0.4$, and $\omega_{c} \approx 0.03$, which are all above the transition point of $\left\langle r_{\omega_{c}}\right\rangle$. In all cases, we compare the spectrum of the bare operator $\mathcal{O}^{\omega_{c}}$ to the distribution $\widetilde{P}_{\omega_{c}}(\lambda)$ of the sign-randomized version $\widetilde{\mathcal{O}}^{\omega_{c}}$; see Eq. (14). As can be clearly seen in Figs. 8(a) and 8(b), $P_{\omega_{c}}(\lambda)$ and $\widetilde{P}_{\omega_{c}}(\lambda)$ differ strongly for the largest $\omega_{c}$ considered. Specifically, while $\widetilde{P}_{\omega_{c}}(\lambda)$ closely follows the semicircle law appropriate for random matrices, $P_{\omega_{c}}(\lambda)$ still exhibits pronounced peaks at $\pm 1 / 2$, which is reminiscent to the original spectrum of the spin-1/2 operator. This deviation between $P_{\omega_{c}}(\lambda)$ and $\widetilde{P}_{\omega_{c}}(\lambda)$ illustrates that the matrix elements $\mathcal{O}_{m n}$ of a small submatrix cannot automatically be considered as
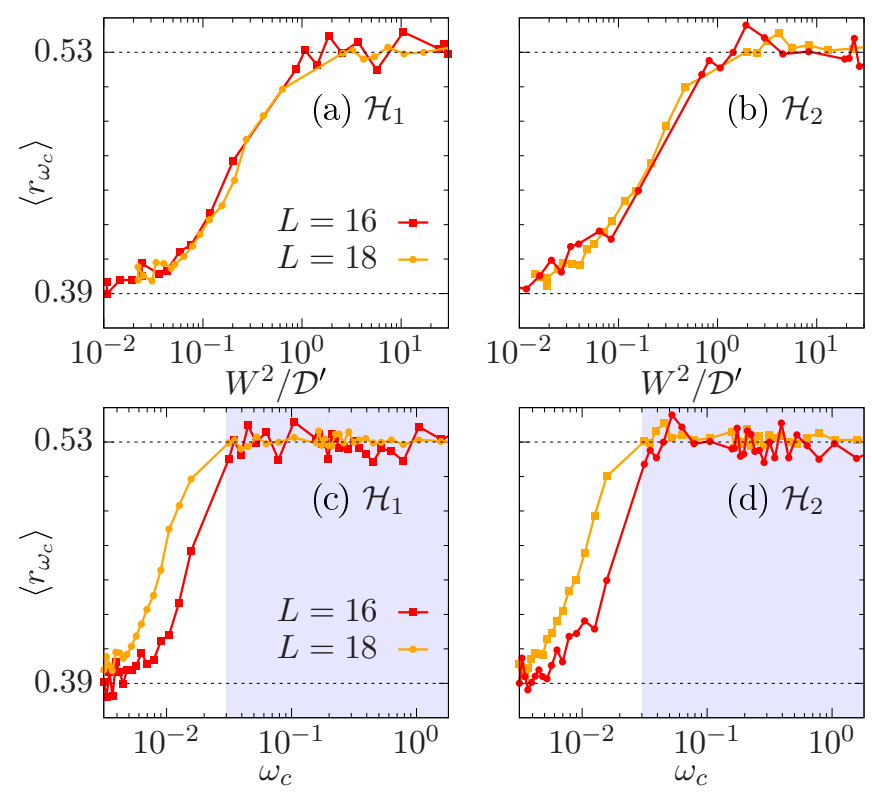

FIG. 7. Mean ratio $\left\langle r_{\omega_{c}}\right\rangle$ of adjacent level spacing of the operator $\mathcal{O}^{\omega_{c}}$ versus [(a),(b)] the scaling parameter $W^{2} / \mathcal{D}^{\prime}$ and $[(\mathrm{c}),(\mathrm{d})]$ the cutoff frequency $\omega_{c}$. Panels (a) and (c) show data for $\mathcal{H}_{1}$ while panels (b) and (d) show data for $\mathcal{H}_{2}$. The data is obtained for system sizes $L=16,18$ and the dashed horizontal lines indicate the GOE value $r_{\mathrm{GOE}} \approx 0.53$ and the Poisson value $r_{\text {Poisson }} \approx 0.39$. For our analysis of $P_{\omega_{c}}(\lambda)$, we restrict ourselves to $\omega_{c} \gtrsim 0.03$ [shaded area in panels (c) and (d)], such that $\left\langle r_{\omega_{c}}\right\rangle \approx r_{\mathrm{GOE}}$.

independent random variables, notwithstanding all standard indicators of ETH being in agreement with the Gaussian distribution. This is a main result of the present paper.

Lowering the cutoff frequency to $\omega_{c} \approx 1,0.4$ and $\omega_{c} \approx$ 0.03 [see Figs. 8(c)-8(h)], we find that the spectra of the bare and the randomized submatrices become more and more similar. Especially for $\mathcal{H}_{1}, P_{\omega_{c}}(\lambda)$, and $\widetilde{P}_{\omega_{c}}(\lambda)$ are very similar for $\omega_{c} \approx 0.4$ and virtually indistinguishable from each other for $\omega_{c} \approx 0.03$. Moreover, the bulk of the spectrum is convincingly described by a semicircular distribution (the width of the semicircle shrinks with $\omega_{c}$ ), while small deviations from a perfect semicircle can be observed at the spectral edges. This similarity of $\widetilde{P}_{\omega_{c}}(\lambda)$ and $P_{\omega_{c}}(\lambda)$ as well as their semicircular shape can be interpreted as an indication that the correlations between the matrix elements are significantly reduced for frequencies around and below $\omega \lesssim \Delta E_{\mathrm{RMT}} \approx 0.4$, i.e., on these smaller scales the $\mathcal{O}_{m n}$ can be represented as independent random variables. This is another central result of the present work. Let us emphasize that the full distribution $P_{\omega_{c}}(\lambda)$ is sensitive to the RMT scale $\Delta E_{\mathrm{RMT}}$ while the mean gap ratio $\left\langle r_{\omega_{c}}\right\rangle$ takes on the GOE value for all $\omega_{c}$ considered in Fig. 8.

Comparing properties of $\mathcal{H}_{1}$ and $\mathcal{H}_{2}$, we find that $P_{\omega_{c}}(\lambda)$ and $\widetilde{P}_{\omega_{c}}(\lambda)$ still differ visibly for $\omega_{c} \approx 0.4$ in the case of $\mathcal{H}_{2}$ [see Fig. 8(f)] but become very similar for the smaller $\omega_{c} \approx 0.03$; see Fig. 8(h). This is in accord with Fig. 9, which suggests that $\Delta E_{\mathrm{RMT}} \approx 0.1$ is smaller in the case of $\mathcal{H}_{2}$.

While it certainly would be desirable to study the eigenvalue distribution $P_{\omega_{c}}(\lambda)$ for even smaller values of $\omega_{c}$ in a controlled manner, this is difficult to do numerically as the 

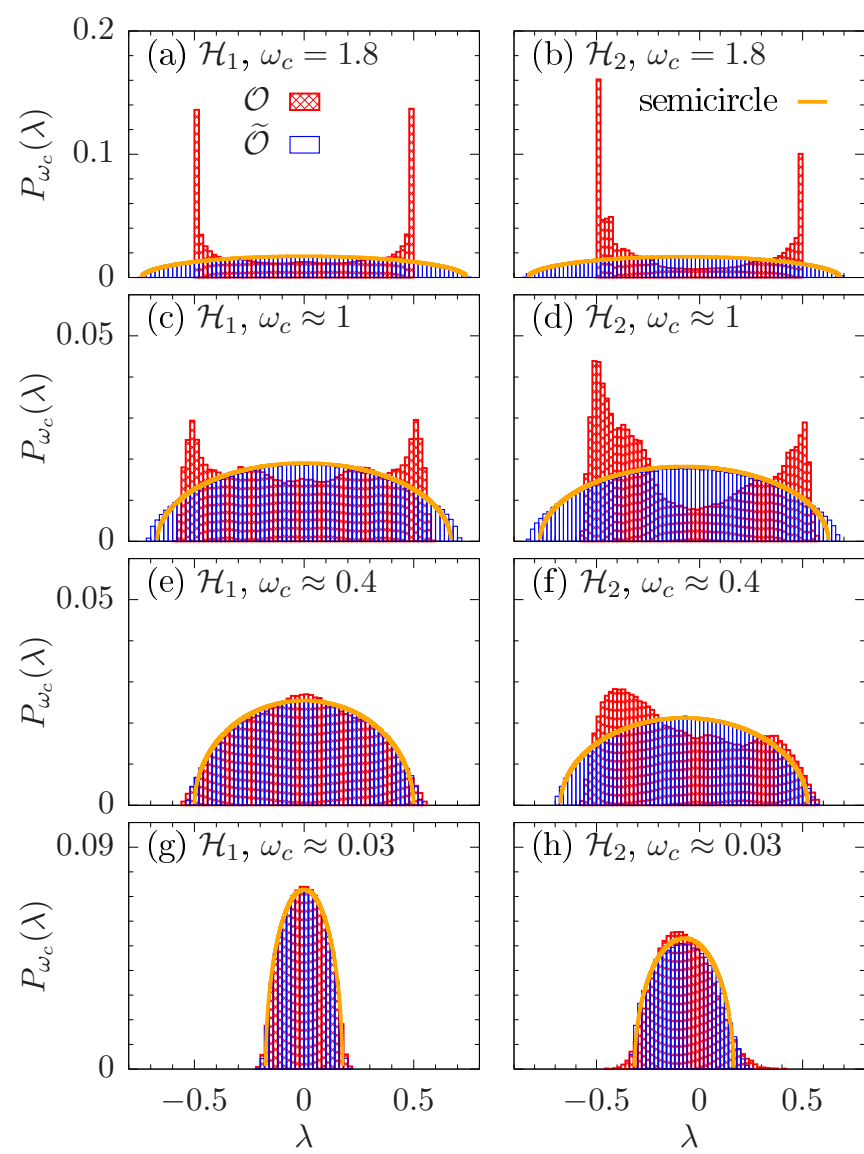

FIG. 8. Eigenvalue distributions $P_{\omega_{c}}(\lambda)$ and $\widetilde{P}_{\omega_{c}}(\lambda)$ of bare and sign-randomized submatrices in the energy window $\bar{E} / L \in$ $[-0.15,-0.05]$ for system size $L=18$. The cutoff frequencies are chosen as $(\mathrm{a}, \mathrm{b}) \omega_{c}=1.8$ (i.e., the full nonband submatrix of dimension $\left.\mathcal{D}^{\prime}<\mathcal{D}\right),[(\mathrm{c}),(\mathrm{d})] \omega_{c} \approx 1,[(\mathrm{e}),(\mathrm{f})] \omega_{c} \approx 0.4$, and $[(\mathrm{g}),(\mathrm{h})]$ $\omega_{c} \approx 0.03$. For comparison, the solid curves indicate a semicircle distribution. Left column shows data for $\mathcal{H}_{1}$, while right column shows data for $\mathcal{H}_{2}$. The skewed distribution in the case of $\mathcal{H}_{2}$ can be explained by the diagonal matrix elements $\mathcal{O}_{m m}$ [see Fig. 3(b)] which have a mean that (i) is nonzero within the energy window and (ii) grows with $E$, in contrast to the case of $\mathcal{H}_{1}$; cf. Fig. 3(a).

value of $W$ and the relative bandwidth size $W / \mathcal{D}^{\prime}$ become too small. Likewise, if one instead diagonalizes full nonband submatrices with smaller dimension $\mathcal{D}^{\prime}$ (see Appendix A), the number of eigenvalues becomes significantly reduced, which complicates the analysis.

Finally, Figs. 9(c) and 9(d) show the difference $d_{2}\left(\omega_{c}\right)$ between the two distributions $P_{\omega_{c}}(\lambda)$ and $\widetilde{P}_{\omega_{c}}(\lambda)$. Consistent with our previous observation in Fig. 8, we find that $d_{2}\left(\omega_{c}\right)$ decreases upon reducing $\omega_{c}$. Moreover, the decrease is slower in the case of $\mathcal{H}_{2}$. The minimum of $d_{2}\left(\omega_{c}\right)$ is reached around the values of $\omega_{c}$ which we associate with $\Delta E_{\mathrm{RMT}}\left(\Delta E_{\mathrm{RMT}} \approx 0.4\right.$ in case of $\mathcal{H}_{1}$ and $\Delta E_{\mathrm{RMT}} \approx 0.1$ in case of $\left.\mathcal{H}_{2}\right)$, and $d_{2}\left(\omega_{c}\right)$ remains low for smaller $\omega_{c}$.

\section{DISCUSSION}

In this paper we have studied matrix elements of the spin- $1 / 2$ operator $\mathcal{O}=S_{L / 2}^{z}$ in the eigenbasis of two

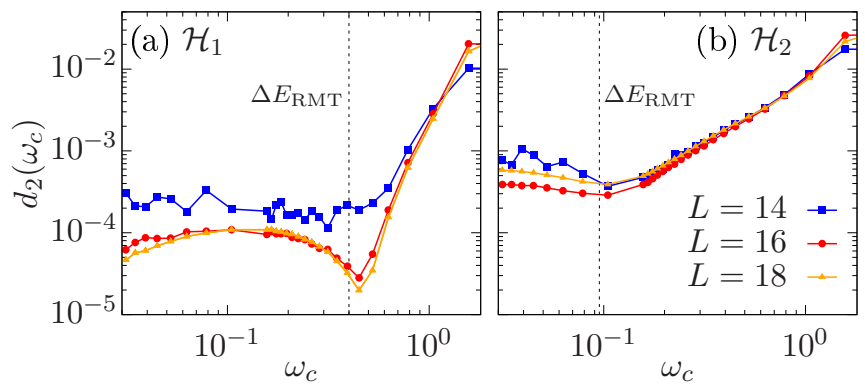

FIG. 9. $d_{2}\left(\omega_{c}\right)$ for (a) $\mathcal{H}_{1}$, and (b) $\mathcal{H}_{2}$. System sizes are chosen as $L=14,16,18$. The dashed vertical lines indicate the approximate location of $\Delta E_{\mathrm{RMT}}$ below which random-matrix behavior occurs.

nonintegrable quantum spin chains. Specifically, we have considered the one-dimensional XXZ model in the presence of two different integrability-breaking perturbations: an additional next-nearest-neighbor interaction and a single-site magnetic field in the center. For these models and an energy window close to the center of the spectrum, we have shown that the matrix elements of $\mathcal{O}$ are in good agreement with the eigenstate thermalization hypothesis ansatz in the sense that (i) variance of the diagonal matrix elements decreases exponentially with the increasing system size, (ii) the offdiagonal matrix elements follow a Gaussian distribution with a variance that depends smoothly on the energy difference $\omega$, and (iii) the ratio between the variances of diagonal and off-diagonal matrix elements approximately takes on the value predicted by random matrix theory. Overall, our results are in full agreement with previous works [29-32] and the conventional expectation that for local operators and nonintegrable Hamiltonians the ETH is satisfied.

The central question of this paper was to study to what extent off-diagonal matrix elements $\mathcal{O}_{m n}$ can be treated as independently drawn random variables. To this end, we have considered submatrices around a fixed mean energy $\bar{E}$ and restricted the $\mathcal{O}_{m n}$ to lie inside a sufficiently narrow band $\left|E_{n}-E_{m}\right| \leqslant \omega_{c}$. We have established the form of the full eigenvalue distribution to be a sensitive probe to correlations between matrix elements. By comparing the eigenvalue distribution of the band submatrix with its sign-randomized counterpart (14), we have shown that the $\mathcal{O}_{m n}$ cannot be considered as independently distributed, even on scales where $\mathcal{O}_{m n}$ follow a Gaussian distribution with a variance that varies comparatively slowly with $\omega$, i.e., on the scales where the ETH function $f(\bar{E}, \omega)$ is approximately constant. Specifically, while the spectrum of the sign-randomized matrix closely followed the semicircle law, matching the theoretical expectation for a random matrix, the eigenvalue distribution of the original submatrix was found to exhibit signatures of the spin operator, implying correlations between the matrix elements. When the cutoff frequency $\omega_{c}$ is sufficiently reduced, we have found that the eigenvalue distribution of the original and the sign-randomized operator become similar and well described by a semicircle. The energy scale $\Delta E_{\mathrm{RMT}}$ when this transition occurs marks the onset of validity of the random-matrix behavior.

It should be noted that many important results rooted in the ETH are not sensitive to the statistics of the off-diagonal 
matrix elements $\mathcal{O}_{m n}$. This includes the central argument that the ETH ensures thermalization [3,6,57], which essentially relies on the exponential smallness of the $\mathcal{O}_{m n}$. At the same time, within the contemporary understanding of ETH, it is often assumed that matrix elements posses additional statistical properties matching the GOE (or some other appropriate Gaussian ensemble); see, e.g., Refs. [3,12,15,21]. In this work, we have advocated that below a certain energy scale all statistical properties of the off-diagonal matrix elements would match those of a Gaussian random matrix. We have provided numerical evidence that this onset of random-matrix behavior takes place below a certain energy scale $\Delta E_{\mathrm{RMT}}$ for specific models and observables. Our results suggest that for frequencies $\omega<\Delta E_{\mathrm{RMT}}$, the notion of (pseudo-)randomness of the $r_{m n}$ entering the ETH can be interpreted in an even stricter sense. At the same time, we have clearly seen that the scale $\Delta E_{\mathrm{RMT}}$, where this transition to genuine random-matrix behavior occurs, is distinctly smaller than the scales on which "standard" indicators of the ETH are fulfilled.

Our work raises a number of straightforward questions. First, we note that our numerical observation $\Delta E_{\mathrm{RMT}} \ll E_{\tau}$ mirrors the analytical bound $\Delta E_{\mathrm{RMT}} \lesssim E_{\tau} / L$ established in Ref. [28], where $E_{\tau}$ is defined as the width of the plateau of $f(\bar{E}, \omega)$ (note that $E_{\tau}$ is sometimes referred to as Thouless energy [38]). A natural question would be to establish the scaling of $\Delta E_{\mathrm{RMT}}$ with the system size $L$ and, in particular, to investigate if $\left(\Delta E_{\mathrm{RMT}}\right)^{-1}$ can be associated with the timescale of late time chaos at which the dynamics of various observables is captured by RMT [58-61].

Another direction is to contrast the behavior in chaotic systems with the integrable counterparts. We repeat the analysis of Sec. III for the integrable XXZ model in the Appendix B. One particular observation to point out is that off-diagonal matrix elements in the integrable case also can be regarded as random and independent, although not Gaussian, below a certain energy scale. We leave for the future the question of better understanding this transition, and the universal properties of $\mathcal{O}_{m n}$ in the integrable case.

Eventually, one avenue of research is to characterize the nature of the correlations between off-diagonal matrix elements for $\omega>\Delta E_{\mathrm{RMT}}$, and to understand their potential impact on self-averaging properties of the $\mathcal{O}_{m n}$ exploited in various works $[39,49,62,63]$. At the same time, it would be interesting to study the connection between universal properties of $\mathcal{O}_{m n}$ at small frequencies and transport, which could be diffusive or ballistic [43].

\section{ACKNOWLEDGMENTS}

We thank M. Bergfeld and M. Lamann for discussions and helpful comments on the manuscript. This work has been funded by the Deutsche Forschungsgemeinschaft (DFG), Grants No. 397107022 (GE 1657/3-1), No. 397067869 (STE 2243/3-1), and No. 355031190, within the DFG Research Unit FOR 2692. A.D. acknowledges support of the Russian Science Foundation (Project No. 17-12-01587).

\section{APPENDIX A: EIGENVALUE DISTRIBUTION OF FULL NONBAND SUBMATRICES}

In Sec. III B we have demonstrated that the eigenvalue distribution of band submatrices approximately takes on a
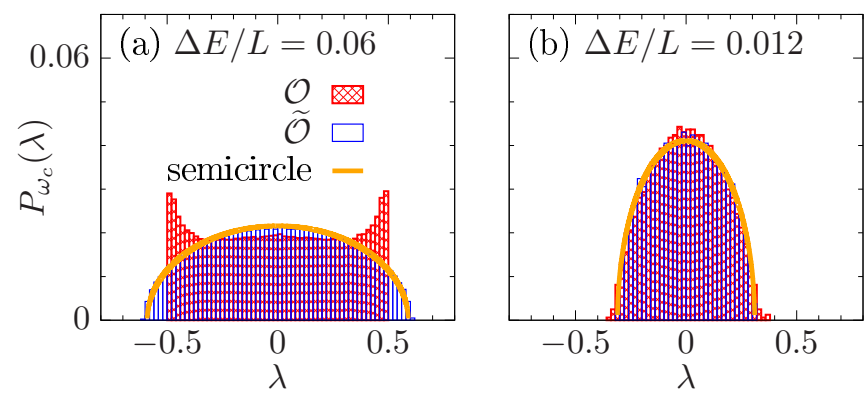

FIG. 10. Eigenvalue distributions $P_{\omega_{c}}(\lambda)$ and $\widetilde{P}_{\omega_{c}}(\lambda)$ for full (nonband) submatrices in the case of the model $\mathcal{H}_{1}$ with $L=18$. The matrices are centered around the mean energy $\bar{E} / L=-0.1$, but the width of the energy window is now smaller compared to the main text, i.e., we choose (a) $\Delta E / L=0.06$ and (b) $\Delta E / L=0.012$, which would correspond cutoff frequencies $\omega_{c} \approx 1$ and $\omega_{c} \approx 0.2$.

semicircle shape when the band is sufficiently narrow. In Fig. 10 we show a qualitatively similar result for the Hamiltonian $\mathcal{H}_{1}$ and full (nonband) submatrices, where the width of the energy window is now chosen as $\Delta E / L \approx 0.06$ and $\Delta E / L \approx 0.012$, i.e., narrower than in the main text. Recall that a smaller $\Delta E$ implies a smaller submatrix dimension $\mathcal{D}^{\prime}$. For the examples shown here, we have $\mathcal{D}^{\prime} \approx 8000$ and $\mathcal{D}^{\prime} \approx 1500$. While $P_{\omega_{c}}(\lambda)$ still exhibits pronounced features at $\lambda= \pm 1 / 2$ for the larger $\Delta E$ in Fig. 10(a), we find that the distributions $P_{\omega_{c}}(\lambda)$ and $\widetilde{P}_{\omega_{c}}(\lambda)$ are essentially indistinguishable for the smaller $\Delta E$ in Fig. 10(b). Moreover, analogous to the results for the band submatrices in Fig. 8, small deviations from a perfect semicircle law appear at the spectral edges if $\Delta E$ is lowered. While we cannot entirely exclude the possibility of finite-size effects, we conclude that our results for band submatrices in the main text, i.e., a semicircular bulk with small deviations at the spectral edges, are not just caused by the finite bandwidth, but are stable features which appear for full nonband matrices as well.

\section{APPENDIX B: RESULTS FOR THE INTEGRABLE MODEL $\mathcal{H}_{\mathrm{Xxz}}$}

In Figs. 3-6 we have analyzed the ETH structure of $S_{L / 2}^{z}$ written in the eigenstates of the two nonintegrable models $\mathcal{H}_{1}$ and $\mathcal{H}_{2}$. In Fig. 11 we present analogous data for the integrable model $\mathcal{H}_{\mathrm{XXZ}}$. As expected, the results for $\mathcal{H}_{\mathrm{XXZ}}$ are drastically different compared with the nonintegrable systems $\mathcal{H}_{1,2}$. In particular, (i) the width of the distribution of the diagonal matrix elements does not visibly shrink with increasing $L$, (ii) $\Gamma(\omega) \neq \pi / 2$ and is nonconstant and dependent on $L$, in agreement with [18], (iii) the ratio $\Sigma^{2}(n, \mu)$ is orders of magnitude larger compared to $\Sigma_{\mathrm{GOE}}^{2}=2$, and (iv) the distribution $P\left(\mathcal{O}_{m n}\right)$ is clearly non-Gaussian (see also Refs. $[12,18])$. Overall, these results confirm the expectation that the ETH is not satisfied in the case of integrable models. The only quantity which exhibits a similar behavior for $\mathcal{H}_{\mathrm{XXZ}}$ and $\mathcal{H}_{1,2}$ is the running average $\overline{\left|\mathcal{O}_{m n}\right|^{2}}$ shown in Fig. 11(b). Namely, we find that plotting $\overline{\left|\mathcal{O}_{m n}\right|^{2}}$ versus $\omega$ for system sizes $L=14,16,18$ yields smooth curves which collapse onto each other when rescaled by the respective Hilbert-space 

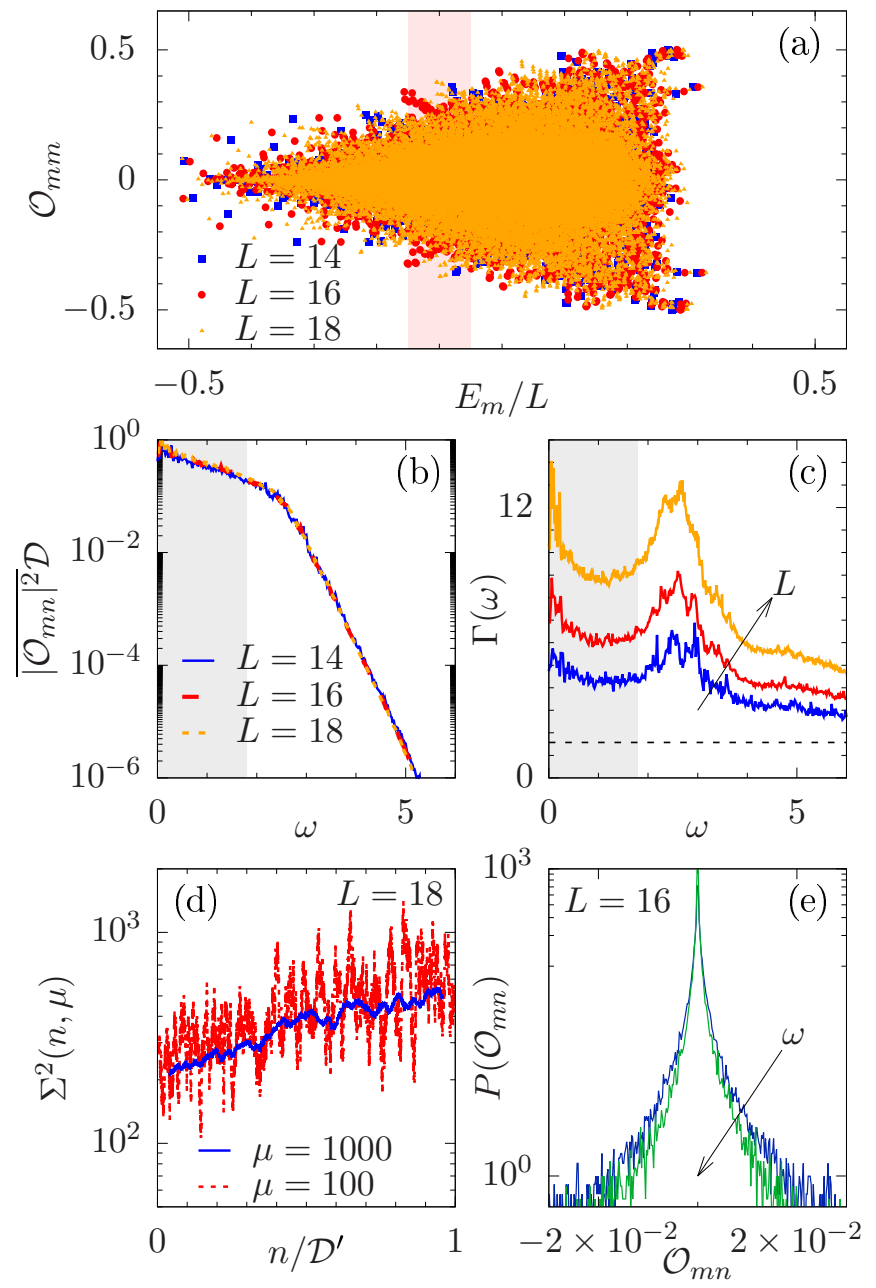

FIG. 11. Data for the integrable model $\mathcal{H}_{\mathrm{XXZ}}$, analogous to the results for $\mathcal{H}_{1,2}$ in Figs. 3-6. (a) Diagonal matrix elements $\mathcal{O}_{m m}$ versus $E_{m} / L$. (b, c) $\overline{\left|\mathcal{O}_{m n}\right|^{2}} \mathcal{D}$ and $\Gamma(\omega)$ for $L=14,16,18$. (d) $\Sigma^{2}(n, \mu)$ for $L=18$ and $\mu=100,1000$. (e) Distribution $P\left(\mathcal{O}_{m n}\right)$ of offdiagonal matrix elements with $\omega=0.1,2$ (arrow). Data in panels (b)-(e) are obtained in the energy window $\bar{E} / L \in[-0.15,-0.05]$.

dimension $\mathcal{D}$. This behavior is in agreement with the recent studies in Refs. [18,64].

Finally, we consider eigenvalue distribution for the band submatrices in the case of integrable model $\mathcal{H}_{\mathrm{XXZ}}$. Figure 12(a) shows results for the level-spacing ratio $\left\langle r_{\omega_{c}}\right\rangle$, while Fig. 12(b) shows the eigenvalue distributions $P_{\omega_{c}}(\lambda)$ and $\widetilde{P}_{\omega_{c}}(\lambda)$ of the original and the randomized band submatrices with the cutoff frequency $\omega_{c} \approx 0.1$. Comparing with the results for the nonintegrable models $\mathcal{H}_{1,2}$, the qualitative behavior of both $\left\langle r_{\omega_{c}}\right\rangle$ and $P_{\omega_{c}}(\lambda)$ appears to be very similar. Namely, we find that $\left\langle r_{\omega_{c}}\right\rangle$ exhibits a crossover from $r_{\mathrm{GOE}}$ to $r_{\text {Poisson }}$ when the cutoff frequency $\omega_{c}$ (or bandwidth $W$ ) decreases. Furthermore, $P_{\omega_{c}}(\lambda)$ and $\widetilde{P}_{\omega_{c}}(\lambda)$ have the very similar shape for the considered value of $\omega_{c}$, which indicates that $\mathcal{O}_{m n}$ within the corresponding band can be considered as independent. Accordingly, as discussed in Appendix C, eigenvalue distribution is approximately semicircular. Given these results, we conclude that mutual independence of the off-diagonal matrix elements below certain energy scale
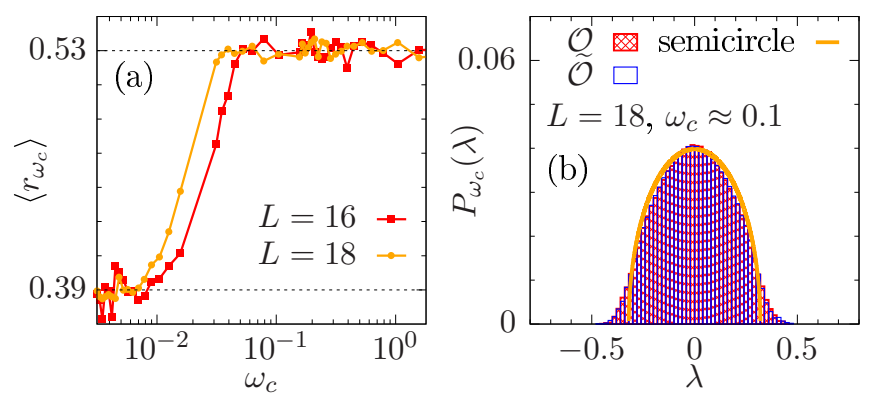

FIG. 12. Properties of eigenvalues of band submatrices for the integrable model $\mathcal{H}_{\mathrm{Xxz}}$. Analogous to the results presented in the main text, the energy window is chosen as $\bar{E} / L \in[-0.15,-0.05]$. (a) $\left\langle r_{\omega_{c}}\right\rangle$ for $L=16,18$. (b) $P_{\omega_{c}}(\lambda)$ and $\widetilde{P}_{\omega_{c}}(\lambda)$ for $L=18$ and $\omega_{c} \approx 0.1$.

$\omega<\Delta E_{\mathrm{RMT}}$ is also present in the integrable models, raising the question if an appropriate non-Gaussian random matrix theory can capture the universal properties of the $\mathcal{O}_{m n}$ in this case.

\section{APPENDIX C: EIGENVALUE DISTRIBUTION OF BAND RANDOM MATRICES}

In this section we briefly review the work of Molchanov et al. [51], which derives an integral equation satisfied by the eigenvalue distribution function of a band random matrix. Namely, we consider an $\mathcal{D} \times \mathcal{D}$ matrix

$$
\mathcal{O}_{n m}=\frac{v(t)}{\sqrt{\mathcal{D}}} r_{n m}, \quad t=(n-m) / \mathcal{D},
$$

where $v$ is a piece-wise continuous function and $r_{n m}$ are independently distributed random variables with zero mean and unit variance. Notice that $r_{n m}$ do not have to be Gaussian, it is sufficient that all $r_{n m}$ are drawn from the same distribution. So far band submatrices of local operators considered in this paper (11) are small enough such that the density of states is approximately constant, they can be modeled by the random matrix $(\mathrm{C} 1)$ with

$$
v^{2}(\omega / \Delta E)=\left\{\begin{array}{cl}
(\Delta E) f^{2}(\bar{E}, \omega), & |\omega| \leqslant \omega_{c} \\
0, & |\omega|>\omega_{c} .
\end{array}\right.
$$

where $\Delta E=E_{\mathcal{D}}-E_{1}$. The resolvent

$$
r(z)=\operatorname{Tr} \frac{1}{z-\mathcal{O}}
$$

can be expressed in terms of an auxiliary function $r(t, z),|t| \leqslant$ $1 / 2$ which satisfies

$$
\begin{gathered}
r(z)=\int_{-1 / 2}^{1 / 2} r(t, z) d t \\
r(t, z)\left[z+\int_{-1 / 2}^{1 / 2} v^{2}\left(t-t^{\prime}\right) r\left(t^{\prime}, z\right) d t^{\prime}\right]+1=0 .
\end{gathered}
$$

There are two limiting cases which can be solved analytically, square random matrix with $v^{2}=$ const (this is the case of $\left.\omega_{c}=\Delta E\right)$ and infinitely narrow band matrix $v^{2}(t)=v_{0}^{2} \delta(t)$ 
(this is the case of $\left.\omega_{c} \ll \Delta E\right)$. In both cases $r(t, z)=r(z)$ is $t$-independent and satisfies $v_{0}^{2} r^{2}+z r+1=0$ where

$$
\begin{gathered}
v_{0}^{2}=\int_{-1 / 2}^{1 / 2} v^{2}(t) d t=2 \int_{0}^{\bar{\omega}} f^{2}(\bar{E}, \omega) d \omega, \\
\bar{\omega}=\min \left(\omega_{c}, \Delta E / 2\right) .
\end{gathered}
$$

The eigenvalue distribution is then the semicircle of radius $2 v_{0}$,

$$
P(\lambda)=\lim _{\epsilon \rightarrow 0^{+}} \frac{1}{\pi} \Im[r(\lambda+i \epsilon)]=\frac{\sqrt{4 v_{0}^{2}-\lambda^{2}}}{2 \pi v_{0}^{2}} .
$$

[1] A. Polkovnikov, K. Sengupta, A. Silva, and M. Vengalattore, Rev. Mod. Phys. 83, 863 (2011).

[2] C. Gogolin and J. Eisert, Rep. Prog. Phys. 79, 056001 (2016).

[3] L. D'Alessio, Y. Kafri, A. Polkovnikov, and M. Rigol, Adv. Phys. 65, 239 (2016).

[4] J. M. Deutsch, Phys. Rev. A 43, 2046 (1991).

[5] M. Srednicki, Phys. Rev. E 50, 888 (1994).

[6] M. Rigol, V. Dunjko, and M. Olshanii, Nature (London) 452, 854 (2008).

[7] M. Srednicki, J. Phys. A 32, 1163 (1999).

[8] M. Feingold and A. Peres, Phys. Rev. A 34, 591 (1986).

[9] M. Feingold, D. M. Leitner, and M. Wilkinson, Phys. Rev. Lett. 66, 986 (1991).

[10] L. F. Santos and M. Rigol, Phys. Rev. E 82, 031130 (2010).

[11] W. Beugeling, R. Moessner, and M. Haque, Phys. Rev. E 89, 042112 (2014).

[12] W. Beugeling, R. Moessner, and M. Haque, Phys. Rev. E 91, 012144 (2015).

[13] H. Kim, T. N. Ikeda, and D. A. Huse, Phys. Rev. E 90, 052105 (2014).

[14] R. Mondaini, K. R. Fratus, M. Srednicki, and M. Rigol, Phys. Rev. E 93, 032104 (2016).

[15] R. Mondaini and M. Rigol, Phys. Rev. E 96, 012157 (2017).

[16] D. Jansen, J. Stolpp, L. Vidmar, and F. Heidrich-Meisner, Phys. Rev. B 99, 155130 (2019).

[17] R. Steinigeweg, J. Herbrych, and P. Prelovšek, Phys. Rev. E 87, 012118 (2013).

[18] T. LeBlond, K. Mallayya, L. Vidmar, and M. Rigol, Phys. Rev. E 100, 062134 (2019).

[19] F. H. L. Essler and M. Fagotti, J. Stat. Mech. (2016) 064002.

[20] R. Nandkishore and D. A. Huse, Annu. Rev. Condens. Matter Phys. 6, 15 (2015).

[21] D. J. Luitz and Y. Bar Lev, Phys. Rev. Lett. 117, 170404 (2016).

[22] N. Shiraishi and T. Mori, Phys. Rev. Lett. 119, 030601 (2017).

[23] C. J. Turner, A. A. Michailidis, D. A. Abanin, M. Serbyn, and Z. Papić, Nat. Phys. 14, 745 (2018).

[24] O. Bohigas, M. J. Giannoni, and C. Schmit, Phys. Rev. Lett. 52, 1 (1984).

[25] L. Foini and J. Kurchan, Phys. Rev. E 99, 042139 (2019).

[26] A. Chan, A. De Luca, and J. T. Chalker, Phys. Rev. Lett. 122, 220601 (2019).

[27] C. Murthy and M. Srednicki, Phys. Rev. Lett. 123, 230606 (2019).

[28] A. Dymarsky, arXiv:1804.08626 (2018).

[29] M. Brenes, T. LeBlond, J. Goold, and M. Rigol, Phys. Rev. Lett. 125, 070605 (2020).

[30] M. Brenes, J. Goold, and M. Rigol, Phys. Rev. B 102, 075127 (2020).
[31] M. Pandey, P. W. Claeys, D. K. Campbell, A. Polkovnikov, and D. Sels, arXiv:2004.05043 (2020).

[32] L. F. Santos, F. Pérez-Bernal, and E. J. Torres-Herrera, Phys. Rev. Research 2, 043034 (2020).

[33] M. Rigol and L. F. Santos, Phys. Rev. A 82, 011604(R) (2010).

[34] J. Richter, F. Jin, H. De Raedt, K. Michielsen, J. Gemmer, and R. Steinigeweg, Phys. Rev. B 97, 174430 (2018).

[35] L. Santos, J. Phys. A 37, 4723 (2004).

[36] O. S. Barišić, P. Prelovšek, A. Metavitsiadis, and X. Zotos, Phys. Rev. B 80, 125118 (2009).

[37] V. Alba, Phys. Rev. B 91, 155123 (2015).

[38] M. Serbyn, Z. Papić, and D. A. Abanin, Phys. Rev. B 96, 104201 (2017).

[39] J. Richter, J. Gemmer, and R. Steinigeweg, Phys. Rev. E 99, 050104(R) (2019).

[40] The variable $n$ indicates the midpoint of the interval, i.e., the sums run from $n-\mu / 2$ to $n+\mu / 2$. In the case of $\sigma_{\text {od }}^{2}(n, \mu)$, the sum over diagonal matrix elements has to be replaced by a double sum over off-diagonal matrix elements instead. Since $\Sigma^{2}(n, \mu)$ can be dependent on the specific values of $n$ and $\mu$, we calculate $\Sigma^{2}(n, \mu)$ for different choices of $\mu$ and all possible intervals $n \in\left[1+\mu / 2, \mathcal{D}^{\prime}-\mu / 2\right]$ which can be embedded along the diagonal of the submatrix.

[41] A. Dymarsky and H. Liu, Phys. Rev. E 99, 010102(R) (2019).

[42] M. L. Mehta, Random Matrices (Elsevier, Amsterdam, 2004).

[43] B. Bertini, F. Heidrich-Meisner, C. Karrasch, T. Prosen, R. Steinigeweg, and M. Žnidarič, arXiv:2003.03334 (2020).

[44] G. Casati, L. Molinari, and F. Izrailev, Phys. Rev. Lett. 64, 1851 (1990).

[45] Y. V. Fyodorov and A. D. Mirlin, Phys. Rev. Lett. 67, 2405 (1991).

[46] M. Kuś, M. Lewenstein, and F. Haake, Phys. Rev. A 44, 2800 (1991).

[47] Y. V. Fyodorov, O. A. Chubykalo, F. M. Izrailev, and G. Casati, Phys. Rev. Lett. 76, 1603 (1996).

[48] F. Borgonovi, F. M. Izrailev, L. F. Santos, and V. G. Zelevinsky, Phys. Rep. 626, 1 (2016).

[49] L. Dabelow and P. Reimann, Phys. Rev. Lett. 124, 120602 (2020).

[50] A. Dymarsky, Phys. Rev. B 99, 224302 (2019).

[51] S. Molchanov, L. Pastur, and A. Khorunzhii, Theor. Math. Phys. 90, 108 (1992).

[52] V. Oganesyan and D. A. Huse, Phys. Rev. B 75, 155111 (2007).

[53] D. Cohen and T. Kottos, Phys. Rev. E 63, 036203 (2001).

[54] T. Kottos and D. Cohen, Phys. Rev. E 64, 065202(R) (2001).

[55] D. A. Abanin, W. De Roeck, and F. Huveneers, Phys. Rev. Lett. 115, 256803 (2015).

[56] A. Avdoshkin and A. Dymarsky, arXiv:1911.09672 (2019). 
[57] M. Rigol and M. Srednicki, Phys. Rev. Lett. 108, 110601 (2012).

[58] J. Cotler, N. Hunter-Jones, J. Liu, and B. Yoshida, J. High Energy Phys. 11 (2017) 48.

[59] J. Cotler and N. Hunter-Jones, arXiv:1911.02026 (2019).

[60] S. Moudgalya, T. Devakul, C. W. von Keyserlingk, and S. L. Sondhi, Phys. Rev. B 99, 094312 (2019).
[61] M. Schiulaz, E. J. Torres-Herrera, and L. F. Santos, Phys. Rev. B 99, 174313 (2019).

[62] C. Nation and D. Porras, Phys. Rev. E 99, 052139 (2019).

[63] J. Richter, F. Jin, L. Knipschild, H. De Raedt, K. Michielsen, J. Gemmer, and R. Steinigeweg, Phys. Rev. E 101, 062133 (2020).

[64] K. Mallayya and M. Rigol, Phys. Rev. Lett. 123, 240603 (2019). 